\title{
Cronología del movimiento feminista en Chile 2006-2016
}

\author{
Silvia Lamadrid Alvarez ${ }^{1}$ (iD) 0000-0002-4770-8570 \\ Alexandra Benitt Navarrete ${ }^{2}$ (iD 0000-0002-551 1-3059 \\ 'Universidad de Chile, Facultad de Ciencias Sociales, Departamento de Sociología, \\ Santiago, Chile. departamento.sociologia@facso.cl \\ 2Universidad de Valparaíso. Instituto de Sociología, Facultad de Humanidades, \\ Valparaíso, Chile.
}

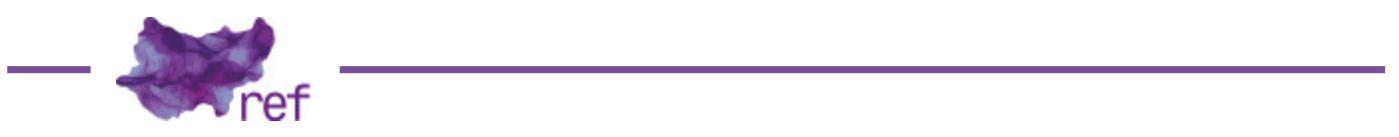

Resumen: En el contexto de una reactivación de los movimientos sociales, el feminismo en Chile ha adquirido creciente protagonismo en el espacio público, con una diversidad de corrientes y organizaciones que intentan articularse en torno a la definición de sus demandas como movimiento social, considerando el debate entre autonomía e institucionalización. Basadas en una línea de tiempo virtual que documenta las principales movilizaciones públicas feministas en el período 2007-2016, pretendemos describir las principales características de ese proceso y analizar los principales nudos de la acción colectiva feminista en el país, evidenciando el emergente protagonismo de las nuevas generaciones desde organizaciones universitarias, propiciando una progresiva masividad en las movilizaciones emblemáticas, pero con moderada efectividad política en la esfera institucional.

Palabras clave: feminismo; acción colectiva; movimiento social

\section{Chronology of the feminist movement in Chile 2006-2016}

Abstract: In the context of a revival of social movements, feminism in Chile has gained increasing prominence in the public space, with a diversity of currents and organizations that try to articulate around the definition of their demands as a social movement, considering the debate between autonomy and institutionalization. Based on a virtual timeline documenting the main feminist public mobilizations in the period 2007-2016, we intend to describe the main characteristics of this process and analyze the main nodes of collective feminist action in the country, highlighting the emerging role of the new generations from university organizations, favoring an enormous growth in the emblematic mobilizations, but with moderate political effectiveness in the institutional sphere.

Keywords: Feminism; Collective action; Social movements

\section{Cronologia do movimento feminista no Chile 2006-2016}

Resumo: No contexto de uma reativação dos movimentos sociais, o feminismo no Chile tem adquirido crescente protagonismo no espaço público, com uma diversidade de correntes e organizações que tentam articular-se em torno à definição de suas demandas como movimento social, considerando o debate entre autonomia e institucionalização. Baseadas em uma linha do tempo virtual que documenta as principais mobilizações públicas feministas no período 2007-2016, pretendemos descrever as principais características deste processo e analisar os principais nós da ação coletiva feminista no país, evidenciando o emergente protagonismo das novas gerações a partir de organizações universitárias, que propiciam um crescimento massivo nas mobilizações emblemáticas, porém com moderada efetividade política na esfera institucional.

Palavras-chave: feminismo; ação coletiva; movimento social 


\section{Introducción}

En los inicios del siglo XXI, en América Latina, ha reaparecido el protagonismo de los movimientos sociales considerados de nuevo tipo, diferentes de los que caracterizaron al siglo XX, sustentados en la identidad de clase y apuntando a reivindicaciones económicas y sociales. Estos movimientos han puesto en cuestión los aparentemente exitosos modelos de transición democrática, develando las tensiones sociales que la globalización y el neoliberalismo habían ido acumulando.

Durante la dictadura cívico militar (1973-1989) hubo un importante movimiento de mujeres, demandando la recuperación de los derechos civiles, políticos y sociales conculcados, y un núcleo crítico a la situación específica de las mujeres, golpeadas por las crisis económicas, que desmoronaron el orden familiar estructurado en torno al padre proveedor. Esas organizaciones de mujeres estuvieron ligadas a los partidos políticos, existiendo una permanente tensión entre aquellas que enfatizaban la centralidad de las demandas feministas y la necesidad de organizaciones autónomas, y quienes validaban las demandas de las mujeres, pero subordinadas a la lucha por la recuperación de la democracia y con ello a la conducción política partidaria (Julieta KIRKWOOD, 1986).

Los gobiernos post dictadura en Chile entre 1990 y 2006, encabezados por la "Concertación de Partidos por la Democracia", mantuvieron y legitimaron el modelo económico y la institucionalidad heredada, reformando los aspectos más autoritarios. La dictadura chilena había implantado más temprano que en otros países de la región, y en forma más completa, por la profundidad de la represión, el nuevo modelo económico, una institucionalidad autoritaria, y remodeló incluso las relaciones sociales, marcándolas con una nueva subjetividad, en que el principal elemento de integración social era el mercado. Frank Gaudichaud (2014) sostiene que el país experimentaba un "progresismo neoliberal o social-liberal maduro". Pero el modelo asentado exitosamente empezó a tener síntomas de agotamiento en el nuevo siglo, en la medida que se visibilizaba la enorme desigualdad social que acompaña la concentración de la riqueza, y la incapacidad del mercado de dar solución a las demandas sociales acumuladas.

En Chile la reactivación de la acción colectiva ha sido encabezada por los movimientos estudiantiles y por nuevos actores y demandas: los movimientos territoriales, ambientalistas, por la diversidad sexual y nuevas organizaciones feministas, cuya presencia ha revitalizado las corrientes que sobrevivían desde los años 80 . Se ha estudiado la situación del feminismo en los 90 , e incluso los primeros años del nuevo siglo, explicando la desarticulación del movimiento feminista en ese período como producto de cambios de la estructura de oportunidades políticas durante la transición, que llevó a la fragmentación del feminismo en dos corrientes: una institucional, que se involucró con el diseño y aplicación de políticas públicas desde el Estado hacia las mujeres, y otra tendencia autonomista, que rechazaba la cooptación del movimiento por los grupos del poder, y buscaba desarrollar una concepción de la acción política y cultural del feminismo fuera de las constricciones de la acción del Estado. Pese a esta tensión, se han incorporado demandas a la agenda política del Estado, paradójicamente, con una paulatina ausencia del movimiento en la esfera pública (Marcela RíOS; Lorena GODOY; Elizabeth GUERRERO, Elizabeth, 2003).

Para distinguir la acción colectiva propiamente feminista de la del movimiento amplio de mujeres, en este trabajo utilizaremos la definición de movimiento de mujeres como:

Todo el espectro de personas que actúan de manera individual, pero también a organizaciones o grupos que están trabajando para aminorar aspectos de la subordinación de género basada en el sexo (...) Algunas partes [del movimiento de mujeres] pueden estar en desacuerdo entre sí, o pueden diferir en sus prioridades, y algunas de sus corrientes, grupos o individualidades pueden permanecer letárgicas durante cierto tiempo. Algunas personas se definen a sí mismas como feministas; otras, probablemente nunca utilizarán esa palabra, pero todas ellas promoverán en sus actividades causas en pro de las mujeres. (Geertje LYCKLAMA à Nijeholt, citada por INSTRAW, 2005 apud Rosa COBO, 2009)

Y nos referiremos a movimiento feminista como "Movimientos sociales y políticos que se fundan en la conciencia de que las mujeres (como colectivo humano) han sido oprimidas, explotadas y dominadas por el patriarcado, en sus diferentes etapas históricas" (COBO, 2009, p. 180).

El período analizado se abre con la elección, por primera vez en Chile, de una mujer para la Presidencia de la República, y se cierra durante el segundo período de Bachelet, con un intermedio del primer gobierno de derecha desde el fin de la dictadura.

Para varias autoras (Claudia MORA; RÍOS, 2009; Teresa VALDÉS, 2010), el mandato de la primera mujer presidenta abría una nueva estructura de oportunidades para la reemergencia de un movimiento feminista, que había entrado en un período de silencio (RÍOS; GODOY; GUERRERO, 2003), y avanzar en demandas estancadas por la falta de voluntad política y la inexistencia de presión eficaz desde la sociedad civil. Se esperaba que el nuevo gobierno impulsara una agenda más progresista, respondiendo a las críticas desde las organizaciones feministas autónomas y de académicas feministas (Kathya ARAUJO, 2009; VALDÉS, 2010) hacia la gestión gubernamental, 
cuestionando su real beneficio hacia los intereses de las mujeres, y señalando su servicio a los intereses del modelo económico neoliberal, que necesitaba una mayor provisión de mano de obra. Otras dudaban de la posibilidad de impulsar cambios significativos sin contar con el apoyo de un fuerte movimiento social (Susan FRANCESCHET, 2006).

La aparición de un masivo movimiento estudiantil por demandas educacionales en el primer año del nuevo gobierno abrió un nuevo período político, gatillando la expresión de otras demandas pendientes en la sociedad chilena, dentro del cual la acción colectiva feminista ha ido escalando en su masividad y radicalidad. En este trabajo nos proponemos describir las principales características de ese proceso, y analizar los principales nudos de la acción colectiva feminista en el país, evaluando su capacidad de articular demandas y gestionarlas políticamente.

Postulamos que el emergente protagonismo de las nuevas generaciones, muchas veces vinculadas al activismo estudiantil, le ha dado una progresiva masividad en la escena pública, pero mantiene una articulación todavía incipiente, limitando su efectividad política para lograr cambios en la esfera institucional, entendiendo por ella fundamentalmente la institucionalidad gubernamental. Eso se vincula tanto con las tensiones entre las tendencias dentro del debate entre autonomía e institucionalización, como con las particulares formas de expresión del feminismo, que logran la organización coyuntural de los diversos grupos, sin conseguir, hasta ahora, articulaciones más permanentes y estructuradas, y enfatizan elementos culturales sobre la articulación y negociación política de sus demandas.

Nos hemos basado en la investigación documental cronológica contenida en la Línea de Tiempo "Movimiento Mujeres en Chile"*, de elaboración propia, consistente en una recopilación de archivos online, principalmente noticias de diversos medios de comunicación sobre manifestaciones públicas de mujeres, feministas y de organizaciones de la disidencia sexual, acontecidas en Santiago y Valparaíso. La línea cuenta con una categorización de los acontecimientos en "Hitos", "Personajes", "Campañas", "Hechos Relevantes", "Manifestaciones Públicas", "Personajes", "Encuentros", "Organizaciones", "Publicaciones" y "Arte" que caracterizan las principales actividades feministas en el espacio público.

Presentaremos primero el contexto político, enfatizando las agendas de género de los respectivos gobiernos. Luego señalaremos los resultados obtenidos con la información utilizada, para analizarlos y exponer las principales conclusiones.

\section{Contexto del período}

Después de tres períodos de gobierno, la coalición Concertación de Partidos por la Democracia experimentaba un creciente desapego entre el electorado. Ya Ricardo Lagos, en 1999, fue elegido en segunda vuelta, a diferencia de los dos primeros presidentes de la Concertación, que obtuvieron mayoría en la primera vuelta. En el 2005, Bachelet también fue elegida en segunda vuelta. Pese a ello, había muchas expectativas en relación con la nueva presidenta. La primera candidatura femenina de la coalición respondía a la percepción de demandas por cambios tras 15 años en el gobierno y su deseo de beneficiarse de que, por su menor presencia en la arena política, las mujeres suelen ser percibidas como menos involucradas en las redes de corrupción (FRANCESCHET, 2006). A ello sumaba otras características atípicas: socialista, agnóstica, soltera, alejada del estereotipo esperable.

Como señalábamos, los gobiernos de la Concertación avanzaron en el reconocimiento de las mujeres como sujetos de derechos, poniendo al día leyes referidas a las relaciones de género en la sociedad chilena. Modificaciones en las leyes de filiación y de matrimonio, permitiendo el divorcio, junto con leyes contra la violencia doméstica y la despenalización de la sodomía fueron promulgadas dentro de acuerdos consensuados entre los dos grandes bloques políticos, con escasa participación de la sociedad civil (Ximena VALDÉS, 2007). Igualmente, la participación de las mujeres en la fuerza laboral continuó creciendo, aunque en el mismo contexto de precariedad laboral.

Diversas autoras (T. VALDÉS, 2010; FRANCESCHET, 2006; María Isabel MATAMALA, 2010; Raquel OLEA, 2010) coinciden en el enorme peso simbólico que tuvo su llegada a la primera magistratura, más allá de las transformaciones que pudiera realizar su gobierno. En un país conservador como Chile, el imaginario tradicional sobre las identidades y relaciones entre hombres y mujeres quedaba trizado; la pregunta era respecto a cuánto y cómo se iba a desarrollar esa fisura.

Sus principales compromisos de campaña apuntaban a mejorar la situación de los sectores de la población que habían sido ajenos a los beneficios del crecimiento económico de las dos últimas décadas, mediante el crecimiento de los servicios de salud y educación, especialmente focalizados en servicios de cuidados a los hijos de mujeres trabajadoras, así como la creación de un código contra las discriminaciones que las afectaban como tales.

Algunas analistas relativizaban la potencialidad transformadora de Bachelet en materia de género, señalando que los avances para las mujeres dependían de la capacidad de los movimientos feministas de superar la fragmentación en que se encontraban, y construir alianzas 
en torno a demandas que les dieran mayor eficacia en la transformación del orden de género (MORA; RÍOS, 2009). Franceschet (2006), por su parte, consideraba que el declinante activismo feminista no permitía esperar una fuerte presión desde el movimiento social que apoyara las transformaciones comprometidas, y dudaba de la real profundidad de esos cambios, ya que el programa no cuestionaba la estrategia económica neoliberal, basal en las discriminaciones laborales hacia las mujeres, y estimaba débil la voluntad política de la mandataria para cumplir con su propia agenda de género.

El nuevo mandato se inició con un gesto importante: la conformación de un gabinete inicial con paridad de género, que no pudo mantener, aunque conservó una proporción de mujeres mayor que cualquier gobierno anterior (Teresa CÁCERES, 2010).

Además de la equidad de género, enfatizó en la focalización social, ampliando la Red de Protección Social para las familias más pobres, para lo cual creó al Consejo Asesor Presidencial para la Reforma Previsional (CP) y al Consejo Asesor Presidencial para la Reforma de las Políticas de la Infancia (Cl).

Una de sus primeras medidas fue la creación de una Comisión de reforma al sistema electoral, incluyendo en sus metas garantizar el equilibrio de género (CÁCERES, 2010), pero el proyecto enviado al Congreso no incluyó medidas positivas para incorporar equitativamente a las mujeres al sistema electoral.

El hecho más significativo durante el gobierno de Bachelet fue la irrupción, en 2006, del masivo movimiento de estudiantes secundarios, que repuso la voz de los actores sociales y cambió la agenda gubernamental. Provocaron el primer cambio de gabinete e incentivaron la presentación de un proyecto de ley general de educación por parte del gobierno. El resultado de ello, la Nueva Ley General de Educación —-Ley № 20.370- no cumpliría con las demandas del movimiento, sobre todo la prohibición del lucro con recursos públicos, que quedaría pendiente.

A pesar de las movilizaciones estudiantiles, las críticas por la implementación del nuevo sistema de transporte colectivo en Santiago, el permanente desafío del movimiento mapuche y los problemas económicos al final del mandato, Bachelet terminó su gobierno con una aprobación de $84 \%$, según la encuesta Adimark. Para algunas autoras (T. VALDÉS, 2010; María de los Ángeles FERNÁNDEZ, 2011 ) Bachelet "desató procesos sociales y culturales en un escenario de crisis de los partidos políticos y agotamiento de la coalición de gobierno cuando la economía mundial tambaleó y en América Latina se inauguraron cambios políticos y sociales" (FERNÁNDEZ 2011 , 248-9), iniciando cambios significativos en la política y en la cultura, particularmente para la condición y situación de las mujeres.

Una visión menos optimista tiene Linda Stevenson (2012), señalando que los resultados prácticos de su gobierno indican que ella expresó menos apoyo y fue menos exitosa en las áreas de derechos más radicales de las mujeres, y Matamala (2010), quien subraya la omisión de aspectos centrales en la transformación del orden de género, como la libertad de las mujeres a decidir sobre sus cuerpos, eludiendo la necesidad de terminar con los obstáculos para el ejercicio pleno de los derechos sexuales y reproductivos, y renunciando a abrir el debate sobre la legislación sobre el aborto, limitando la corporalidad a la violencia física y sexual, el derecho a la educación de las mujeres adolescentes embarazadas y madres, y el derecho a la atención de salud sin discriminaciones. La otra omisión clave es la del trabajo no remunerado; el interés se centró en trabajo que se transa en el mercado, validando la jornada parcial de las mujeres y su responsabilidad respecto al cuidado de los hijos, reforzando "el rol tradicional de las mujeres como responsables exclusivas del trabajo doméstico, a pesar de su incorporación al mercado laboral, donde su rol sería complementario" (MATAMALA, 2010, p. 150).

A pesar del alto apoyo a la presidenta, en las siguientes elecciones fue elegido el candidato de la derecha por un estrecho margen. Las explicaciones de este fracaso electoral, para Augusto Varas (2010), están en factores como "el agotamiento del proyecto concertacionista, el déûcit intelectual y ético asociados al mismo, así como factores más estructurales propios del sistema político chileno como su crisis de representación y los límites redistributivos" (NARAS, 2010, p.10).

Para este autor, la pérdida del voto de las mujeres se debió a que las expectativas de las mujeres se habían desplegado durante el gobierno de Bachelet, mientras las propuestas de la candidatura de Frei eran muy limitadas e improvisadas respecto a problemas claves para las mujeres, como las tensiones entre familia y trabajo.

Por su parte, Castiglioni (2010) señala además la creciente desafección política del electorado, que había llevado al envejecimiento del padrón electoral y el aumento de la abstención, ante lo cual la concertación no supo interpretar el deseo de cambio, presentando un candidato de continuidad, el expresidente Frei. El discurso de Sebastián Piñera enfatizando la necesidad de la alternancia en el poder, para dar espacio a nuevos actores en el gobierno, resultó más atractivo.

Para Gaudichaud (2015), este primer gobierno de derecha, democráticamente elegido desde 1958, pretendía significar una posible renovación política y una inflexión en el proceso de transición, gobernando directamente para la clase empresarial y administrando el Estado con el 
modelo de gestión de sus empresas. Pero lo que realmente caracterizó el período fue la irrupción de los movimientos sociales con una masividad suficiente para impactar el campo político del gobierno y los partidos, así como a la opinión pública.

Las promesas de cambio del nuevo gobierno apuntaban a reformas políticas para mejorar la calidad de la democracia y corregir el sistema de representación, buscando superar la desconfianza en las instituciones políticas que se había instalado. Hacia el fin de su período, Octavio Avendaño (2013) señala que se habían aprobado los proyectos de inscripción automática y voto voluntario y de realización de elecciones primarias voluntarias dentro de los partidos, quedando pendientes otras reformas comprometidas (fundamentalmente las propuestas destinadas a modernizar el Estado y mejorar la gestión pública), y habiendo omitido tanto la reforma al sistema electoral binominal como la regulación del financiamiento de la política.

El gobierno de Piñera no acogió las propuestas negociadas entre la oposición y el oficialismo como efecto de las movilizaciones sociales del año 201 1, ni menos las demandas de la sociedad civil para abrir aspectos claves del ordenamiento constitucional a nuevas formas de participación, manteniendo la coherencia con las concepciones sobre democracia de la derecha chilena (AVENDAÑO, 2013). En cambio, en varias políticas sectoriales, como las de educación, salud y seguridad ciudadana, el gobierno tuvo que alterar su agenda para poder responder a las demandas sociales. Desde inicios de 2011, el gobierno se vio desafiado por movilizaciones regionales, ambientales y sobre todo estudiantiles, todas con alto costo político, tanto para la imagen del presidente, cuya aprobación descendió al $23 \%$, como para el funcionamiento, ya que llevaron a constantes renuncias de ministros y obligándolo a recurrir a parlamentarios en ejercicio para esos cargos.

Para Avendaño (2013), estos conflictos sociales visibilizaron las limitaciones de la institucionalidad para resolverlos; tampoco los partidos de la oposición, la Concertación, tuvieron la capacidad de elaborar propuestas alternativas ante esas demandas, de modo que la opinión pública castigó a los partidos en general, tanto de gobierno como de oposición.

Manuel Antonio Garretón (2016) estima que las movilizaciones de 2011 y 2012 abrieron un nuevo ciclo, porque sus demandas plantearon la necesidad de un nuevo proyecto histórico, partiendo por los problemas educacionales, pero extendiéndose a todos los ámbitos de la vida social, economía, institucionalidad, cultura y relaciones internacionales. Ello planteó la urgencia de una nueva propuesta de cambio, que la Concertación, transformada en Nueva Mayoría con la integración del Partido Comunista, intentó presentar en las elecciones presidenciales de 2013.

En cuanto a la agenda de género del gobierno Piñera, Pamela Díaz-Romero (2010) sostiene que los contenidos del programa de gobierno de Sebastián Piñera

evidencian una concepción tradicional del orden de género, combinada con las ideas neoliberales que anteponen el rol del mercado a la responsabilidad que le cabe al Estado en reconocer y garantizar el ejercicio de los derechos. (DÍAZ-ROMERO, 2010, p.147)

Desde el primer año del gobierno de Piñera, las feministas comprobaban sus temores respecto de las políticas públicas de género, al poner el centro de su política no en las mujeres como sujetos de derechos, sino en una familia concebida sin contradicciones internas, en que la complementariedad tradicional de género funciona armónicamente, y que no correspondía a la realidad de las diversas familias chilenas. Las políticas se focalizaron en las mujeres de sectores denominados vulnerables, priorizando la creación de empleo para ellas, entendiendo que sus problemas son producto de la pobreza y no de las relaciones de género. Ello implicó reajustar la institucionalidad estatal creada por la Concertación para proponer y coordinar las políticas de equidad de género, despolitizando el Servicio Nacional de la Mujer (Carmen TORRES, 2011).

En coherencia con este enfoque, y en respuesta a demandas desde el área de la salud, se extendió el permiso postnatal de 3 a 6 meses, asegurando 24 semanas de descanso completo para las madres. También se entregaron bonos para la contratación de mujeres de menores ingresos, se creó el "Programa 4 a 7 . Mujer trabaja tranquila", para dar apoyo después de la jornada escolar a los hijos o menores a cargo de mujeres trabajadoras, y se dio capacitación específica para la inserción laboral femenina en la actividad minera.

La candidatura de Bachelet en 2013, aunque triunfante en segunda vuelta, no logró remontar la crisis de representación, puesto que fue elegida con 120.000 votos menos que en 2005 (GAUDICHAUD, 2015). Y, al igual que en ese año, su victoria se sostuvo en la figura de la candidata como encarnación de la imagen de la madre protectora, consensual y apelando a la solidaridad de género, confrontada a la candidata de la derecha, también mujer e hija de un general de la dictadura, pero con una imagen ruda y agresiva, representando ambas dos modelos de mujer y los dos lados en la disputa por los Derechos Humanos. Además, ella acababa de dejar la Presidencia de ONU-Mujer.

Dentro del contexto latinoamericano, Garretón (2017) sostiene que, mientras en otros países de la región las transiciones postdicatura intentaron enfrentar tanto los enclaves autoritarios heredados como las consecuencias de la globalización y las reformas neoliberales, Chile no 
había dado este paso, y sería el segundo gobierno de Michelle Bachelet el que presentaría el primer proyecto refundacional del período post dictatorial.

Desde esa posición y sosteniéndose en equilibrios políticos complejos dentro de su coalición, y escuchando aparentemente las demandas de los movimientos sociales, Bachelet presentó una agenda de reformas sustanciales, con tres ejes centrales: una reforma constitucional democratizadora y participativa, segundo, una reforma fiscal que tasara los beneficios enormes de las grandes empresas, y una reforma de la educación para terminar con el copago y el lucro y avanzar hacia la gratuidad en 6 años.

Las reformas más importantes fueron morigeradas, relativizando su impacto en el modelo neoliberal, por la presión ejercida por los partidos de derecha, como expresión política, apoyada por el duopolio mediático, las Iglesias y los gremios y asociaciones patronales. Pero fueron suficientes para confundir a los movimientos sociales, que no tuvieron la capacidad de rearticular su agenda ante la ofensiva reformista, "Oscilando entre integración en las instancias de 'participación' del gobierno y deseo de movilizar a sus bases, sin lograrlo realmente" (GAUDICHAUD, 2015, p. 70).

En materia de equidad de género, Bachelet propuso dar un mayor nivel institucional a las instancias enfocadas en ello, creando el Ministerio de la Mujer y la Equidad de Género (proyecto de ley promulgado el 8 de marzo de 2015) y enviando un proyecto de Ley de Cuotas para estimular la inclusión de mujeres en las candidaturas a los cargos de representación popular (ya promulgado). Rompiendo el tradicional dominio en este cargo del Partido Demócrata Cristiano, nombró ministra a la militante comunista Claudia Pascual. A cambio, el primer gabinete ya no intentó la paridad: sólo 9 de 23 ministros fueron mujeres, menos del $40 \%$.

Para el tercer año de Bachelet, la ONG Ciudadano Inteligente estimaba en $68 \%$ el cumplimiento de sus promesas electorales, incluyendo la ley de cuotas, el proyecto para modificar la legislación de violencia intrafamiliar, aumentando las penas y ampliando la concepción de violencia más allá de la relación de pareja o la familia, y sobre todo por la presentación del proyecto de despenalización de la interrupción voluntaria del embarazo en tres causales, peligro de la vida de la madre, violación o inviabilidad del feto, aprobado finalmente en agosto de 2017.

En suma, el contexto político estuvo fuertemente marcado por el agotamiento del modelo político de los consensos, que administró la institucionalidad heredada de la dictadura, y la emergencia de los movimientos sociales que expresaban el descontento con los nudos más críticos. La agenda de género estuvo marcada por el énfasis en las políticas de apoyo a la integración al mercado de trabajo, especialmente de las mujeres de los llamados sectores vulnerables, y la lucha contra la violencia intrafamiliar. Ya sea con la reivindicación discursiva del apoyo al empoderamiento y los derechos de las mujeres por Bachelet, o con la perspectiva familista de Piñera, el centro de las políticas apuntaba a la incorporación de la mano de obra femenina a la fuerza de trabajo y al rechazo a la violencia hacia las mujeres. Sólo en el último período de Bachelet se retoman las demandas de promoción de participación política (paridad) y de derechos reproductivos (aborto por tres causales).

\section{Los debates dentro del feminismo}

A continuación, revisaremos los debates al interior del feminismo latinoamericano y chileno, en tanto nos permiten comprender las tendencias que se expresan en la particular conformación de la acción colectiva feminista en el período revisado.

El movimiento feminista internacional ya desde los años '70 se ha visto interpelado por la emergencia de nuevos sujetos políticos que tensionaron las prácticas y discursividades centradas en el sujeto universal mujer. El feminismo negro, lésbico y trans han permeado en las teorizaciones de diversas corrientes que resignifican el quehacer político feminista desde la necesidad de incluir el concepto de diversidad.

Las críticas dentro del feminismo latinoamericano han estado centradas en la hegemonía de un discurso personificado por mujeres de élite, blancas, heterosexuales y de sectores urbanos, crítica transversal al movimiento internacional, pero en el caso latinoamericano se extiende además a la razón colonialista que perdura en los discursos dominantes del movimiento, sujeta a un tránsito de conocimientos con dependencia epistemológica de sur a norte (María Luisa FEMENÍAS, 2007: Yuderkys ESPINOSA, 2009), desconociendo el contexto geopolítico que lo sitúa. En este debate, el feminismo latinoamericano ha intentado recoger la especificidad de la diversidad en su propio contexto histórico, utilizando y transformando su influencia en un marco de interpretación que evidenciaba al sistema patriarcal de las sociedades latinoamericanas mestizas e indígenas, en alianza a una moral catolicista (ARAUJO, 2008; Francesca GARGALLO, 2006).

Desde los años setenta, y recuperando la herencia del movimiento sufragista experimentado por gran parte de la región, el movimiento se activó para enfrentar los gobiernos autoritarios y dictatoriales, con luchas enfocadas en los derechos humanos y la recuperación de la democracia. Sin embargo, ya desde el Primer Encuentro Feminista Latinoamericano, en 1981, se evidenció la división entre un feminismo de izquierda y feministas autónomas (GARGALLO, 2006). 
Estas divisiones tendrán una nueva dimensión al momento de negociar los procesos democráticos, donde acontece una fuerte institucionalización del movimiento feminista, produciendo conflictos intramovimiento, que tuvieron su punto de inflexión en el VI Encuentro Feminista Latinoamericano y del Caribe en 1993 y se intensificaron en el siguiente Encuentro en Cartagena en 1996, llevando al fraccionamiento entre autónomas e institucionales, con el protagonismo de sectores lesbofeministas en el primer sector (ESPINOSA, 2009: ARAUJO, 2008). Los sectores institucionales no sólo estuvieron protagonizados por feministas en partidos políticos o cargos públicos, sino también por el rol que las mismas sostuvieron desde la academia, donde se instaló rápidamente la categoría de género como concepto para interpretar las relaciones sociales latinoamericanas. Sobre estos antecedentes, se construyen los principales ejes del pensamiento feminista latinoamericano, sostenidos por una ética de la justicia social, los derechos humanos e intrínsecamente ligada a la experiencia de las mujeres.

Reflejo del movimiento a nivel latinoamericano, el feminismo nacional tuvo como punto de inflexión la dictadura pinochetista, desde el cual nace una corriente importante de posicionamiento autonomista bajo el lema "Democracia en el país, en la casa y en la cama", que repercutió en todo el territorio. Sin embargo, una vez llegado el tránsito a la democracia bajo la "política de los acuerdos" establecida entre los conglomerados de la época, supuso la fragmentación del bloque feminista que se enfrentó a la dictadura entre "autónomas" e "institucionales" desencadenando en un nuevo "silencio feminista" durante los años '90 (Nicole FORSTENZER, 2013). Este silencio no implicó una ausencia de organizaciones o manifestaciones públicas, sino una búsqueda de unidad bajo una identidad feminista, y luego, una desarticulación del movimiento por diferencias respecto de los espacios políticos y las estrategias de movilización para, a finales de la década, perder protagonismo público y desarticular el repertorio de demandas (RíOS et al., 2003). Durante este período el quehacer feminista adquiere un carácter institucionalizado, manifestado por una proliferación de ONG's y la incidencia de estudios de género en los círculos académicos, ambas aristas situando como protagonista al sujeto mujer, pero careciendo de un cuestionamiento sustantivo sobre el mismo (Verónica FELIÚ, 2009).

Desde la década del 2000 se articuló fuertemente en espacios académicos una corriente feminista de disidencia sexual, cuestionando los esencialismos, apelando a la deconstrucción del sistema sexo/género, y a todas las relaciones de dominación que procuren algún tipo de determinación sobre los individuos, resignificando instituciones sociales como el trabajo, la educación y la medicina. En el caso latinoamericano, la recepción y apropiación crítica de los conceptos y teorías posmodernas internacionales constituyen una homogeneización de diferentes facetas políticas, bajo el concepto queer o postfeminista, disminuyendo el potencial crítico de los mismos en su cruce con la realidad latinoamericana (Felipe RIVAS, 2011). Así, nuevas corrientes, como el feminismo postcolonial y los feminismos comunitarios, también posicionan la etnia y la raza como elementos fundamentales para explicar la configuración del sujeto y el quehacer político feminista latinoamericano (ESPINOSA, 2009).

\section{Las principales expresiones del proceso de movilización feminista}

Una de las señas de identidad de las organizaciones de mujeres la ha constituido la conmemoración de fechas que corresponden a acuerdos internacionales referidos a demandas surgidas de los movimientos de mujeres internacionales y latinoamericanos, en momentos distintos: Día Internacional de la Mujer, Día por la Despenalización del Aborto y Día Contra la Violencia Hacia las Mujeres.

El 8 de marzo de 2006 estuvo signado por la reciente elección de una mujer en la Presidencia, y más de un centenar de organizaciones sociales y no gubernamentales convocaron a un acto en Alameda con Namur, con una concurrencia significativa, aunque no hay números aproximados (OBSERVATORIO GÉNERO Y SALUD, 2006b). En enero ya se había registrado una funa contra el femicida de Victoria Solis, convocada por Red Chilena contra la violencia doméstica y sexual (OBSERVATORIO GÉNERO Y SALUD, 2006a); luego, una reunión feminista en el Centro Cultural de España, y la conmemoración del Día por la Despenalización del Aborto, afirmando la autonomía de las mujeres y rechazando la intromisión de la Iglesia Católica. En septiembre se puso en primera línea la causa feminista, cuando la justicia ratificó la decisión del gobierno de distribuir la píldora del día después de forma gratuita y sin necesidad del consentimiento de tutores legales para jóvenes entre 14 y 18 años.

Este hecho provocó una fuerte reacción por parte de los sectores conservadores, que recurrieron al Tribunal Constitucional para impedirlo. Finalmente, y luego de un 2007 con escasas expresiones públicas feministas, la declaración de inconstitucionalidad de la distribución del fármaco con el argumento de que no existían estudios científicos suficientes que comprobaran que éste no impedía la anidación del óvulo ya fecundado en el útero provocó una marcha masiva en reacción con más de 15.000 participantes (Daniela ESTRADA, 2008), mayoritariamente jóvenes. Finalmente, se decretó su distribución nuevamente el 2010, pero las manifestaciones 
apoyando esta medida continuaron hasta el 2013, cuando el MINSAL oficializó la entrega del PAE a menores de 14 años (Vanessa VARGAS, 2014).

Los derechos reproductivos han sido parte del repertorio habitual de acciones feministas, y adquirieron protagonismo en las diferentes movilizaciones feministas anuales, como "El Día por la Despenalización del Aborto" (28 de septiembre), las movilizaciones por un "Aborto Libre, Seguro y Gratuito" (25 de julio) y el "Día Internacional de Acción por la Salud de las Mujeres" (28 de mayo). En el contexto de ésta última, se lanzó la campaña "Miso pa' Todas" (2016), realizada por la Red Feminista de Entrega de Información para un "Aborto Libre, Seguro y Autónomo", conformada por diecinueve colectivas feministas (RED CHILENA CONTRA LA VIOLENCIA HACIA LA MUJER, 2016), entre las que destacan la Línea de Aborto Libre, Nosotras Decidimos y Colectivo Tijeras, que buscan visibilizar y democratizar el acceso a la información sobre métodos de interrupción del embarazo (FONDO ALQUIMIA, 2017).

La movilización por un "Aborto Libre, Seguro y Gratuito" del 25 Julio de 2013 fue una de las manifestaciones simbólicamente más relevantes para el feminismo chileno y sobre todo santiaguino. Esta marcha contó con una adherencia estimada de 3.000 personas, según Emol (2013), pero de 10.000 según los manifestantes (Tabla 1), pero al final de ella de cientos de personas irrumpieron en la Catedral de Santiago, con presencia de feministas autónomas y de la disidencia sexual gritando consignas en contra de la iglesia y a favor del aborto, incluso rayando las dependencias, lo que marcó esta fecha. De ahí en adelante las movilizaciones en esta temática mantuvieron una adhesión constante de 3.000 a 5.000 participantes, hasta el ingreso del Proyecto de Ley de Aborto en tres causales al Congreso (2015), siendo las movilizaciones del 2016 y 2017 las más emblemáticas, con una participación de con 10.000 y 50.000 asistentes respectivamente (Tabla 1), lo que sin duda influyó en la aprobación de la ley en el Congreso y luego de la revisión del Tribunal Constitucional (2017), sin mucha conformidad por parte de las feministas cuya demanda principal es por el aborto libre. Estas últimas fueron organizadas desde la Coordinadora Feministas en Lucha, que articula muchas secretarías y vocalías de universidades, y a partir de 2016 también logra incentivar acciones en regiones para el 25 de julio, aumentando la cantidad de personas que participan y el grado de adhesión a "aborto libre" a nivel nacional.

Otra de las reivindicaciones con repercusión mediática y manifestaciones públicas fue la extensión del postnatal de seis meses, con movilizaciones durante el 2010 , manifestaciones que se articularon en torno al "Movimiento Ciudadano por un Postnatal de 6 Meses Íntegros", con más de 45 organizaciones gremiales, sindicales, profesionales, estudiantiles, de mujeres y ONG's, caracterizado por manifestaciones de mujeres amamantando en grupo en la vía pública. En el año 2011 finalmente se promulgó la ley que amplió el permiso postnatal materno a 5 meses y medio, incluye a trabajadoras temporales con contrato a plazo fijo y traspasa 1 mes y medio de permiso al padre (NACER EN CHILE, 2012).

Respecto del propio quehacer feminista, destaca el Encuentro Feminista en Olmué (2005) con más de 120 participantes, que no propició ninguna articulación concreta, pero donde se expresó la fragmentación de la acción colectiva y el definitivo distanciamiento del Feminismo Autónomo (que tendrá su propio Encuentro Latinoamericano durante el año 2009, en Ciudad de México) (ESPINOSA, 2009). El 2007 se celebró en Chile el VII Encuentro Lésbico Feminista Latinoamericano y Caribeño con más de 200 participantes (Norma MOGROVEJO, 2007), organizada por el Bloque Lésbico, con tensiones respecto del desplazamiento de temáticas como el racismo, nudo feminista que resurge en el caso de la Machi Francisca Linconao, acusada de participar en un incendio y homicidio (2016) en cuyo apoyo el Feminismo Lésbico Antirracista se ha rearticulado y logrado un posicionamiento mediático (RED DE LESBIANAS Y FEMINISTAS, 2017). Causa bastante distante de las sostenidas por el Lesbofeminismo, que ha tenido un papel secundario dentro del repertorio político del movimiento feminista, con demandas enfocadas en la "visibilidad", mediante campañas sostenidas por Rompiendo el Silencio en torno a la salud sexual "Torta no Seas Pastel" (2015) y La lucha por los Derechos Filiativos (ROMPIENDO EL SILENCIO, 2017). Otra arista ha estado protagonizada por la Línea de Aborto Libre, que entrega información segura sobre aborto con medicamentos hasta las 12 semanas de gestación y para la defensa de las mujeres en el caso de verse enfrentadas a situaciones de violencia cultural, médica y/o policial (Ĺ́NEA ABORTO LIBRE, 2017).

Respecto de la Violencia de Género, en el año 2007 se lanzó la Campaña "iCuidado! El Machismo Mata" por la Red Chilena Contra la Violencia Hacia las Mujeres, que denuncia el femicidio, la violencia sexual, simbólica y toda forma de discriminación hacia las mujeres, logrando posicionamiento público e incidiendo en la configuración de la figura del femicidio en la esfera política, teniendo como consecuencia la promulgación de la Ley de Femicidio (2010). El dramático caso de Nabila Riffo, víctima de un femicidio frustrado (2016) que causó revuelo nacional por las cruentas características del suceso (EL MAGALLANEWS, 2016), motivó que ese año la marcha contra la violencia hacia las mujeres congregara de 50.000 a 80.000 adherentes (Tabla 1). Ha sido relevante también la articulación que realiza la Red Chilena contra la Violencia Hacia las Mujeres en torno a la violencia de género, aun cuando ha habido cierta competencia con quienes 
encabezan las movilizaciones de "Ni Una Menos" (2015), marchas que repudian los casos de violencia de género a nivel latinoamericano (Carlos ARIAS, 2015), articulación que en los próximos años termina en manos de organizaciones universitarias con protagonismo de Pan y Rosas. Estas manifestaciones no contienen demandas específicas ni fechas conmemorativas, sino que se articulan en base a la contingencia de ciertos casos de violencia o femicidio que logran protagonismo mediático, desde el cual logran congregar importantes adeptos a través de las redes sociales.

Otra de las reivindicaciones que ha logrado posicionarse desde un efectivo manejo mediático y de las redes sociales es la temática del acoso callejero, causando gran impacto en los últimos 5 años, promoviendo un Proyecto de Ley de Respeto Callejero (2015), con el protagonismo del Observatorio contra el Acoso Callejero (OCAC) como su propulsora. En particular, dentro de la Universidad de Chile se movilizaron las recién creadas secretarías y vocalías de sexualidad y género, como parte de los centros de estudiantes y la misma FECH, por las denuncias de acoso sexual hacia profesores del Departamento de Historia, llegando a paros y tomas de facultad.

Desde otro espectro del feminismo, han surgido los primero Circuitos de Disidencia Sexual (CUDS, 2006) que, desde la crítica a la concepción de género y a la política institucional, generan tensiones tanto con el feminismo tradicional como con el movimiento de diversidad sexual. Este posicionamiento se ha caracterizado por su creativo repertorio de acciones, con intervenciones en las marchas feministas y de la diversidad sexual, como en la Marcha del Orgullo (2006) donde visten de sacerdotes, monjas y papas, criticando el rol de la Iglesia Católica, y repartiendo cajas de fósforos con su consigna: "La única iglesia que ilumina es la que arde" o manifestaciones en relación a la criminalización del aborto con una acción teatral llamada "Desplazamiento de La Moneda" (2014), donde aparecen encapuchadas/os y con fetos, con pancartas que enunciaban "El Derecho a No Nacer" (CUDS, 2006), así como la campaña "Dona por un aborto". En sincronía con el movimiento estudiantil surgen organizaciones de disidencia sexual en establecimientos emblemáticos de educación media, como el Colectivo Lemebel, creado en 2013, que también se manifiesta desde acciones performativas y ha logrado gestionar espacios como el "Festival Contracultural por la Educación No Sexista", con la participación de diferentes referentes del feminismo de la disidencia.

En este contexto, dentro de los cambios y desafíos en los repertorios y demandas del movimiento estudiantil en los últimos años, han sido importantes los atributos que el feminismo le ha aportado al movimiento. Han emergido nuevos espacios de significación y campos políticos, desde la crítica feminista al imaginario patriarcal de los cantos y consignas en las movilizaciones sociales (Kevin NORAMBUENA, 2016), así como una articulación transversal en espacios universitarios respecto del levantamiento de organismos institucionales universitarios como comisiones y secretarías con perspectiva de género desde el año 2012 (Victoria VIÑALS, 2017) que han sido las principales promotoras de demandas internas en las universidades: protocolos para casos de acoso sexual, así como la incorporación en sus repertorios de temáticas de la diversidad o la disidencia sexual.

Tabla № 1. Manifestaciones emblemáticas feministas y cantidad de asistentes estimadas/os en Santiago.

\begin{tabular}{|c|c|c|c|c|c|c|c|c|c|c|}
\hline $\begin{array}{l}\text { Año/ } \\
\text { Evento }\end{array}$ & 2008 & 2009 & 2010 & 2011 & 2012 & 2013 & 2014 & 2015 & 2016 & 2017 \\
\hline $\begin{array}{l}\text { Dia de la } \\
\text { Mujer } \\
8 \text { marzo }\end{array}$ & $\begin{array}{l}\sin \\
\text { dato }\end{array}$ & 3.000 & $\begin{array}{l}\text { sin } \\
\text { dato }\end{array}$ & 500 & 15.000 & "miles" & 50.000 & 12.000 & 9.000 & $\begin{array}{c}30.000 \mathrm{a} \\
40.000\end{array}$ \\
\hline $\begin{array}{c}\text { Marcha } \\
\text { por la } \\
\text { Libertad } \\
\text { de Decidir } \\
25 \text { julio }\end{array}$ & $\begin{array}{l}15.000 \\
\text { (PAE) }\end{array}$ & $\begin{array}{l}\sin \\
\text { dato }\end{array}$ & $\begin{array}{l}\text { sin } \\
\text { dato }\end{array}$ & $\begin{array}{l}\text { sin } \\
\text { dato }\end{array}$ & $\sin$ dato & $\begin{array}{c}3.000^{*} \mathrm{a} \\
10.000\end{array}$ & $\begin{array}{c}2.000 \mathrm{a} \\
5.000\end{array}$ & 2.000 & 10.000 & 15.000 \\
\hline $\begin{array}{c}\text { Dia } \\
\text { Contra la } \\
\text { Violencia } \\
25 \\
\text { noviembre }\end{array}$ & $\begin{array}{l}\text { sin } \\
\text { dato }\end{array}$ & $\begin{array}{l}\sin \\
\text { dato }\end{array}$ & $\begin{array}{l}\text { sin } \\
\text { dato }\end{array}$ & $\begin{array}{l}\text { sin } \\
\text { dato }\end{array}$ & 200 & 700 & $\begin{array}{l}\sin \\
\text { dato }\end{array}$ & "Decenas" & $\begin{array}{c}50.000 \mathrm{a} \\
80.000\end{array}$ & . \\
\hline
\end{tabular}

Fuente: elaboración propia, a partir de información de medios en internet. 


\section{Conclusiones}

El despliegue del nuevo ciclo de movilizaciones feministas no había sido pronosticado ni por las analistas que ponían sus expectativas en las reformas desde el Estado (FERNÁNDEZ, 2011 ; T. VALDÉS, 2010) ni por quienes cuestionaban la posibilidad de esos cambios sin la presión de la acción colectiva de las mujeres (FRANCESCHET, 2006; MATAMALA, 2010; FORSTENZER, 2013). Observamos que la voluntad de hacer cambios era efectivamente débil, como señala Stevenson respecto al primer gobierno de Bachelet, pero que la reacción del movimiento en defensa de la distribución de anticonceptivos por parte del sistema de salud contuvo la ofensiva derechista y marcó el inicio de una presencia sostenida de las demandas feministas durante el período de Piñera, motivando a una mayor potencia en la agenda de género del nuevo gobierno de Bachelet, en 2014.

Este nuevo ciclo de movilizaciones feministas está entrelazado con la activación de toda la sociedad, con los estudiantes como protagonistas emblemáticos. El movimiento estudiantil desde su irrupción en 2006 ha presentado formas nuevas de participación social y política, articulándose en redes masivas y con una lógica de funcionamiento rizomático (Gilles DELEUZE; Félix GUATTARI, 1997), no piramidal, utilizando las nuevas redes sociales y de información que proveen las nuevas tecnologías de la información (OPECH, 2009). Las movilizaciones del 2011 lograron romper con la ilegitimidad de la protesta pública, para instalarla como la forma fundamental de expresión de las demandas ciudadanas. Desplegaron formas creativas de acciones colectivas, con resignificaciones de la cultura de los medios de comunicación, desplazando la política de los espacios institucionales hacia la sociedad misma (GAUDICHAUD, 2014).

En el caso del feminismo, su reactivación tiene un carácter generacional muy marcado, vinculado a la extensión de los servicios educacionales a sectores cada vez más amplios de jóvenes. El movimiento estudiantil ha tenido un componente femenino mayor que los históricos movimientos reformistas universitarios, tanto en sus bases como, por primera vez, en sus dirigencias. Desde mitad de los años 90 numerosas profesoras feministas han instalado cursos y centros de investigación de género en las Universidades de Chile, Concepción, Academia de Humanismo Cristiano y varias otras, donde han recibido formación en esta perspectiva un número considerable de estudiantes.

Muchos colectivos feministas han emergido dentro del movimiento estudiantil, porque las jóvenes, y también los jóvenes empiezan a plantear sus problemas específicos y validar la diversidad del movimiento, distinta a la clásica identidad masculina y de clase media. A su vez el feminismo ha aportado a los cambios y desafíos que ha experimentado el movimiento estudiantil en sus repertorios y demandas en los últimos años. Han trascendido nuevos espacios de significación y campos políticos, desde la crítica a los cantos y consignas en las movilizaciones sociales, así como una articulación transversal en espacios universitarios apuntando al levantamiento de organismos institucionales universitarios con perspectiva de género (secretarías, comisiones, etc.) que han sido las principales promotoras de demandas internas en las universidades: protocolos para casos de acoso sexual, así como la incorporación en sus repertorios de temáticas de la diversidad o la disidencia sexual. Es en espacios académicos donde emerge y se sitúa la disidencia sexual, de modo que la proliferación de los discursos postfeministas sin duda se sostiene en el nivel educacional de quienes articulan y promueven la acción colectiva, y tienen acceso a cierto capital cultural, que puede desvincularlo de la alianza con las pobladoras, una de las señas de identidad del movimiento de mujeres en la resistencia dictatorial.

El feminismo chileno tiene una larga historia de acción colectiva, con gran diversidad de experiencias políticas y organizacionales, y de formas de coordinación que están presentes hoy en este ciclo de movilizaciones. Sin duda lo nuevo es la participación juvenil y estudiantil, pero, aunque sea visible la diferencia generacional con el feminismo desarrollado bajo dictadura, en el movimiento actual están participando todas, no sin tensiones, en las marchas y manifestaciones. Las diferencias apuntan, además de la experiencia de vida, al cuestionamiento de las más jóvenes a los compromisos adquiridos por las mayores con la institucionalidad, renunciando a la radicalidad de la propuesta feminista, como al rechazo de las mayores hacia el rupturismo de algunas acciones de las jóvenes, como la toma de la Catedral en 2013. Es decir, hay visiones políticas diversas, construidas en base a sus diferentes prácticas como movimiento.

Esta relación entre ambos movimientos se ha expresado en una renovación y masificación de las discursividades y manifestaciones feministas tradicionales instaladas por el feminismo histórico (Día de la Mujer, Día Contra la Violencia Hacia Las Mujeres, Día por la Salud Sexual y Reproductiva de las Mujeres), así como en la nueva articulación en torno a la contingencia internacional del movimiento "Ni Una Menos".

A las coordinaciones y organizaciones históricas se han sumado una gran variedad de grupos. Parte de ellos son organizaciones autogestionadas, caracterizadas por estar compuestas sólo por mujeres, en tanto las que se vinculan más directamente con espacios universitarios son 
mixtas y se plantean feministas o desde una perspectiva de género, tomando temáticas referentes a las mujeres y a la diversidad o disidencia sexual.

La nueva caracterización del movimiento ha promovido una masificación de los discursos de género en la sociedad civil, estableciéndose discusiones al respecto a nivel mediático y político, provocando un posicionamiento social importante; como consecuencia de aquello, las mismas manifestaciones públicas se han caracterizado por presentar un número importante de asistentes y de apariciones mediáticas, así como publicaciones recurrentes con temáticas de género, sean libros, columnas, fanzines o revistas universitarias. Otra arista de lo anterior se sugiere en relación con la utilización de las redes sociales, los actuales medios de comunicación permiten una articulación, difusión y adherencia inmediata a las convocatorias realizadas por el movimiento, lo que ha provocado una explosión en los números de asistentes a las manifestaciones públicas tradicionales (día de la mujer, salud sexual y reproductiva, aborto). Esta característica es de especial relevancia, en la medida en que permite la articulación y tránsito de demandas feministas o de género a nivel internacional, de manera tal que movilizaciones en Argentina o Brasil repercuten en la agenda políica nacional un símbolo representativo de aquello es el movimiento \#NiUnaMenos, que ha significado un despliegue masivo de manifestaciones públicas bajo el contexto de la violencia de género experimentada por los países de la región.

Ahora bien, aunque exista una importante presencia pública de las acciones colectivas feministas, a la fecha del análisis las manifestaciones no habían desencadenado en la articulación o proposición y posicionamientos de demandas de una manera integral. De esta forma, si bien se ha masificado y establecido un espacio social para las discursividades feministas, no ha tenido la fuerza tal de desembocar en el logro de los objetivos del mismo, ni en generarse nuevas demandas políticas culturales o institucionales. Un caso emblemático en este aspecto es el logro de la despenalización del aborto en tres causales, que no deja conformes a un amplio sector feminista que reivindica el aborto libre. Cabe anotar que esta situación cambia ya durante el año siguiente a nuestro análisis, 2017, en que varios de los partidos políticos del nuevo referente creado para las elecciones presidenciales y parlamentarias de ese año incorporaron en sus discursos demandas feministas e incluso la candidata, Beatriz Sánchez, se definía como feminista. De este modo, es posible esperar a futuro una mayor capacidad de articulación para plantear institucionalmente estas demandas.

En las marchas se observan, por ejemplo, la escasez de consignas comunes, aparte de las convocatorias centrales, constituyéndose en un colectivo híbrido y tolerante, pero sin una conducción colectiva común, más allá de los lienzos que la encabezan. Están empezando a constituirse liderazgos visibles y validados, a partir de las vocerías, tanto del movimiento Ni Una Menos como de las organizaciones estudiantiles. En este período, ese espacio en los medios todavía es ocupado por la ministra de SERNAM, quien quiera que ella sea. Esta dificultad sin duda se relaciona con la diversidad interna, y la falta de espacios de debate y articulación donde se debatan las tensiones y diferencias, derivando en que, en algunos años, ha habido convocatorias paralelas en la marcha más emblemática, la del 8 de marzo de 2011 . Este evento es una de las manifestaciones donde aparece un problema, por demás instalado desde los tiempos de la dictadura, que es la cooptación de ese día por parte de diversas agrupaciones políticas, que, con el pretexto de llenarlo de contenido clasista, lo vacían de contenido feminista, enarbolando consignas sobre derechos sociales comunes a toda la población. Se trata de agregar "mujer" a demandas laborales, sin nada que las identifique como específicas de mujeres. En todo caso, esa utilización tiende a desaparecer en las manifestaciones que tienen un claro sello de reivindicaciones feministas, como la denuncia de la violencia de género y los femicidios, o la demanda por aborto libre.

En suma, junto con relevar la presencia mediática/pública/discursiva que la acción colectiva feminista ha alcanzado, hay que reconocer, sin embargo, las dificultades de articulación que han obstaculizado el desarrollo de una agenda común de negociaciones con la institucionalidad que le den una mayor eficacia política. Ha habido grandes avances y aprendizajes, especialmente en la revalidación del feminismo como actor social y en la legitimación de sus demandas en el espacio público, pero sin duda queda mucho camino por recorrer en el proceso de fortalecer el protagonismo del movimiento feminista y sus propuestas en la democratización real de la sociedad chilena.

\section{Referencias}

ARAUJO, Kathya. "Individuo y Feminismo. Notas desde América Latina". Ícono, Revista de Ciencias Sociales, n. 33, p. 141-153, 2008.

ARAUJO, Kathya. "Los procesos de institucionalización como procesos de recepción: Agendas transnacionales y contextos locales en el caso de Chile. Revista Universum, v. 2, n. 24, p. 12-27, 2009. 
ARIAS, Carlos. "'Ni una menos, ni una muerta más', la marcha que llega a La Moneda para exigir el fin del femicidio". Diario UChile [En línea]. Santiago, 3 jul. 2015. Disponible en http://radio.uchile.cl/ 2015/06/03/ni-una-menos-ni-una-muerta-mas-la-marcha-que-llega-a-la-moneda-para-exigir-elfin-del-femicidio/. Acceso en 20/04/2016.

AVENDAÑO, Octavio. "Las reformas políticas en el gobierno de Sebastián Piñera: Chile, 20102013". Revista Mexicana de Ciencias Políticas y Sociales, n. 218, p. 167-191, 2013.

CÁCERES, Teresa. "¿Tengo que mandar como hombre o puedo hacerlo como yo quisiera?”. In: BUROTTO, Alejandra; TORRES, Carmen (Eds.). Y votamos por ella. Michelle Bachelet: miradas feministas. Santiago: Fundación Instituto de la Mujer-Fundación Heinrich Boll, 2010. p. 35-56.

CASTIGLIONI, Rossana. "Chile y el giro electoral: La vergüenza de haber sido y el dolor de ya no ser”. Revista de Ciencia Política, v. 30, n. 2, p. 231-248, 2010.

COBO, Rosa. "Otro recorrido por las ciencias sociales: género y teoría crítica". In: APARICIO, Marta; LEYRA, Begoña; ORTEGA, Rosario (Eds.). Cuadernos de género: Políticas y acciones de género. Madrid: Universidad Complutense, 2009. p. 11-36.

COORDINADORA UNIVERSITARIA DISIDENCIA SEXUAL (CUDS) "Universitarios Herejes en Marcha del Orgullo Gay/Les/Trans". Blogspot [En línea]. 22 octubre 2006. Disponible en http:// portalcuds.blogspot.cl/. Acceso en 18/05/2016.

DELEUZE, Gilles; GUATTARI, Félix. "Rizoma: Introducción”. In: DELEUZE, Gilles; GUATTARI, Félix. Mil mesetas: Capitalismo y Esquizofrenia. Valencia: Ed. Pre-textos, 1997. p. 9-32.

DÍAZ-ROMERO, Pamela. "La dimensión de género en la administración Piñera. Una nueva forma de gobernar”. Barómetro, p. 142-161, 2010.

EL MAGALLANEWS. "Trasladan a ex Posta Central a mujer brutalmente agredida en Coyhaique". EI Magallanews [En línea]. 17 mayo 2016. Disponible en http://www.elmagallanews.cl/noticia/ sociedad/trasladan-ex-posta-central-mujer-brutalmente-agredida-en-coyhaique. Acceso en 21/ 03/2017.

EMOL. "Marcha en favor del aborto concluyó con destrozos en la Catedral" [En línea]. 26 julio 2013 Disponible en http://www.emol.com/noticias/nacional/2013/07/25/61 1065/marcha-en-favor-delaborto-se-realiza-esta-noche-en-el-centro-de-santiago.html. Acceso en 15/04/2016.

ESPINOSA, Yuderkys. "Ełnocentrismo y Colonialidad en los Feminismos Latinoamericanos: Complicidad y Consolidación de las Hegemonías Feministas en el Espacio Transnacional". Revista Venezolana de Estudios de la Mujer, v. 14, n. 33, p. 37-54, 2009.

ESTRADA, Daniela. "Mujeres-Chile: Multitud exige la píldora del día después". Inter Press Service [En línea]. 22 abril 2008. Disponible en http://www.ipsnoticias.net/2008/04/mujeres-chile-multitud-exigela-pildora-del-dia-despue. Acceso en 22/11/2016.

FELIÚ, Verónica. “¿Es el Chile Post-dictadura Feminista?”. Revista Estudos Feministas, v. 17, n. 3, p. 701-715, 2009.

FEMENÍAS, María Luisa. "Esbozo de un Feminismo Latinoamericano". Revista Estudos Feministas, v. 15, n. 1, p. 11-25, 2007.

FERNÁNDEZ, María de los Ángeles; RUBILAR, Fernando. "En el nombre del género: El caso de Michelle Bachelet”. Revista de Ciências Sociais da PUC-Rio, n. 9, p. 135-156, 2011.

FONDO ALQUIMIA. "Activistas lanzan campaña Miso pa todas, infinitas causales" [En línea]. 21 junio 2017 . Disponible en https://www.fondoalquimia.org/ddssrr/activistas-lanzan-campana-misopa-todas-infinitas-causales/. Acceso en 30/10/2017.

FORSTENZER, Nicole. "Ser feminista en el Chile actual: ambigüedades y dilemas de las reivindicaciones de igualdad de género". Universidad de París [En línea]. 2013 (s.f). Disponible en https://chili-neoliberal.sciencesconf.org/conference/chili-neoliberal/pages/ Forstenzer_Ser_feminista_en_el_Chile_actual_ambiguedades_y_dilemas.pdf. Acceso en 11/07/ 2016.

FRANCESCHET, Susan. "El triunfo de Bachelet y el ascenso político de las mujeres". Nueva Sociedad, n. 202, p. 13-22, 2006. 
GARGALLO, Francesca. Ideas Feministas Latinoamericanas. Historia de las Ideas. México: UACM, 2006.

GARRETÓN, Manuel Antonio. "El proyecto de transformación y la crisis político-institucional de la sociedad chilena. El gobierno de Bachelet entre 2014-2016". In: ARQUEROS, Claudio; IRIARTE, Álvaro (Eds). Chile y América Latina. Crisis de las izquierdas del siglo XXI. Universidad del Desarrollo, 2017. p. 209-244.

GARRETÓN, Manuel Antonio. "Crisis de representación, movilizaciones sociales y elecciones presidenciales 2013 en Chile". In: MAYORGA, F. (Compilador). Elecciones y legitimidad democrática en América Latina. Plural Editores, 2016. p. 15-37.

GAUDICHAUD, Franck. "Progresismo Transformista, Neoliberalismo Maduro y Resistencias Sociales Emergentes". Revista OSAL - CLACSO, n. 35, 2014.

GAUDICHAUD, Franck. Las Fisuras del Neoliberalismo Chileno: Trabajo, Crisis de la 'Democracia Protegida' y Conflictos de Clase. Buenos Aires: CLACSO, 2015.

KIRKWOOD, Julieta. Ser política en Chile. Las feministas y los partidos. Santiago, Chile: Facultad Latinoamericana de Ciencias Sociales. 1986.

LÍNEA ABORTO LIBRE. “Línea Aborto Libre”. [En línea]. 2017. Disponible en http://infoabortochile.org/ ?page_id $=549$.

MATAMALA, María Isabel. "A medio camino en un entrevero: ¿quedó desnuda la igualdad de género?". In: BUROTTO, Alessandra; TORRES, Carmen (Eds.). Y votamos por ella. Michelle Bachelet: miradas feministas. Santiago: Fundación Instituto de la Mujer-Fundación Heinrich Böll, 2010. p. 141-168.

MOGROVEJO, Norma. "VII Encuentro Lésbico Feminista Latinoamericano y del Caribe: Autonomía y Racismo". Blogspot [En línea]. 12 febrero 2007. Disponible en http://normamogrovejo.blogspot.cl/ 2016/02/chile-vii-encuentro-lesbico-feminista.html. Acceso en 21/09/2016.

MORA, Claudia; RÍOS, Marcela. "¿De Política de Representación a Política de Coalición?: Posibilidades de Movilización Feminista en el Chile Post-Dictadura". Polis, v. 8, n. 24, p. 133-145, 2009.

NACER EN CHILE. "Logros y desafíos pendientes de las leyes de protección a las m(p)aternidades en Chile: un poco de historia" [En línea]. Abr. 2012. Disponible en http://nacerenchile.blogspot.cl/ 2012/04/un-poco-de-historia-logros-y-desafios.html\#more.

NORAMBUENA, Kevin. "Estudiantes del Liceo 1 marchan contra el gobierno y cuestionan machismo". La Izquierda Diario [En línea]. 25 mayo 2016. Disponible en https://www.laizquierdadiario.cl/ Estudiantes-del-Liceo-1-marchan-contra-el-gobierno-y-cuestionan-machismo.

OBSERVATORIO DE GÉNERO Y SALUD. "Día Internacional de la Mujer: Nuevos desafíos para el movimiento de mujeres en Chile" [En línea]. 1 mar. 2006b. Disponible en http:// www.observatoriogenerosalud.cl/news.php. Acceso en 01/03/2016.

OBSERVATORIO DE GÉNERO Y SALUD. "Primera 'funa' contra autor de femicidio" [En línea]. 5 ene. 2006a. Disponible en http://www.observatoriogenerosalud.cl/news.php. Acceso en 01/03/2016.

OPECH. De Actores Secundarios a Estudiantes Protagonistas. Santiago: Imprenta Salesianos, 2009.

OLEA, Raquel. "Michelle Bachelet: fases y facetas de su representación pública". In: BUROTTO, Alessandra; TORRES, Carmen (Eds.). Y votamos por ella. Michelle Bachelet: miradas feministas. Santiago: Fundación Heinrich Böll y Fundación Instituto de la Mujer, 2010. p. 17-34.

RED CHILENA CONTRA LA VIOLENCIA HACIA LAS MUJERES. "Campaña Miso pa' Todas" [En linea]. 25 mayo 2016. Disponible en http://www.nomasviolenciacontramujeres.cl/campana-miso-patodas/. Acceso en 11/09/2017.

RED CHILENA CONTRA LA VIOLENCIA HACIA LAS MUJERES. "iCuidado! El Machismo Mata" [En linea]. 2007. Disponible en http://www.nomasviolenciacontramujeres.cl/wp-content/uploads/2015/1 1/ 34171-CARTILLA-FEMICIDIO-web.compressed.pdf. Acceso en 11/09/2017.

RED DE LESBIANAS Y FEMINISTAS ACTIVANDO. "Comunicado de la Red de Lesbianas y feministas: 
activando, informando y exigiendo la libertad de la Machi Francisca Linconao". Mapuexpress [En linea]. Enero 2017. Disponible en http://www.mapuexpress.org/?p=15289. Acceso en 11/09/2017.

RIVAS, Felipe. "Territorios de la Disidencia Sexual: Diga "queer" con la lengua afuera, sobre las confusiones del debate latinoamericano". In: CUDS. Por un Feminismo sin Mujeres: Fragmentos del Segundo Circuito de Disidencia Sexual. Santiago: CUDS, 201 1, p. 59-75.

RÍOS, Marcela; GODOY, Lorena; GUERRERO, Elizabeth. Un Nuevo Silencio Feminista. La Transformación de un Movimiento Social en el Chile Posdictadura. Santiago: CEM y Ed. Cuarto Propio, 2003.

ROMPIENDO EL SILENCIO. "\#Matrimoniolgualitario y derechos de filiación: declaración pública" [En linea]. 26 agosto 2017. Disponible en http://www.rompiendoelsilencio.cl/matrimonioigualitarioy-derechos-de-filiacion-declaracion-publica/. Acceso en 12/09/2017.

STEVENSON, Linda. "The Bachelet effect on gender-equity policies". Latin American Perspectives, v. 39, n. 4, p. 129-144, 2012.

TORRES, Carmen (Coord.). Miradas y Reflexiones Feministas: Sebastián Piñera, Año Uno: Conmociones y Exigencias Sociales. Santiago: Fundación Instituto de la Mujer y Fundación Heinrich Böll Cono Sur, 2011.

VALDÉS, Teresa. "El Chile de Michelle Bachelet ¿Género en el poder?". Latin American Research Review, p. 248-273, 2010.

VALDÉS, Ximena. Notas sobre la metamorfosis de la familia en Chile. 2007. Recuperado el 16 de 10 de 2009, de CEPAL, Serie Seminarios y Conferencias, Santiago. Disponible en https://dds.cepal.org/ eventos/presentaciones/2007/1 122/Resumen.XimenaValdes.pdf. Acceso en 12/08/2016.

VARAS, Augusto. "Michelle Bachelet: la derrota de un éxito". Política Exterior, v. 24, n. 134, p. 133$144,2010$.

VARGAS, Vanessa. "Feministas y píldora del día después: El derecho de los padres es en realidad el derecho de propiedad de los adultos sobre niños y niñas". EI Desconcierto [En liena]. 23 de julio 2014. Disponible en http://www.eldesconcierto.cl/2014/07/23/feministas-y-pildora-del-dia-despuesla-defensa-sobre-el-derecho-de-los-padres-es-en-buenas-cuentas-el-derecho-de-propiedad-delos-adultos-sobre-ninos-y-ninas/. Acceso en 14/09/2016.

VIÑALS, Victoria. "Las políticas universitarias para enfrentar los casos de acoso sexual". Diario Uchile [En linea]. 9 enero 2017. Disponible en http://radio.uchile.cl/2017/01/09/las-politicasuniversitarias-para-enfrentar-los-casos-de-acoso-sexual/. Acceso en 12/09/2017.

Silvia Lamadrid Alvarez (silamadr@u.uchile.cl) es socióloga, P. Universidad Católica de Chile; Doctora en Historia, Universidad de Chile. Académica del Depto. de Sociología de la Facultad de Ciencias Sociales de la Universidad de Chile. Coordinadora del Núcleo de Investigaciones en Género y Sociedad "Julieta Kirkwood". Sus líneas de investigación son la sociología e historia de los géneros y los movimientos feministas.

Alexandra Benitt Navarrete (alexandra.benitt@gmail.com) es socióloga de la Universidad de Valparaíso, Chile. Cursando un Magíster en Estudios de la Imagen de la Universidad Alberto Hurtado, Chile. Ha participado en proyectos de investigación vinculados al género, la migración y actualmente, sobre la memoria y los espacios nostálgicos en la ciudad.

\section{COMO CITAR ESTE ARTíCULO, DE ACUERDO CON LAS NORMAS DE LA REVISTA:}

ALVAREZ, Silvia Lamadrid; NAVARRETE, Alexandra Benitt. "Cronología del movimiento feminista en Chile 2006-2016”. Revista Estudos Feministas, Florianópolis, v. 27, n. 3, e54709, 2019.

\section{CONTRIBUCIÓN DE AUTORÍA}

Silvia Lamadrid Alvarez: Concepción, análisis de datos, elaboración del manuscrito, redacción y discusión de resultados.

Alexandra Benitt Navarrrete: Recolección de datos, análisis de datos, colaboración en la redacción. 


\section{FINANCIACIÓN}

Programa de Apoyo a la Productividad Académica, PROA VID 2014; Universidad de Chile (Academic Productivity Support Program, PROA VID 2014; University of Chile).

\section{CONSENTIMIENTO DE USO DE IMAGEM}

No se aplica

\section{APROBACIÓN DE COMITÉ DE ÉTICA EN INVESTIGACIÓN}

No se aplica

\section{CONFLICTO DE INTERESSES}

No existe conflito de intereses

\section{LICENCIA DE USO}

Este artigo está licenciado sob a Licença Creative Commons CC-BY Internacional. Com essa licença você pode compartilhar, adaptar, criar para qualquer fim, desde que atribua a autoria da obra.

\section{HISTÓRICO}

Recebido em 31/12/2017

Reapresentado em 21/11/2018

Aprovado em 27/03/2019 